\title{
Ultrassonografia testicular no modo color Doppler/espectral e avaliação andrológica de garanhões tratados com gonadotrofina coriônica humana (hCG) em diferentes estações do ano
}

[Testicular ultrasonography in the doppler / spectral color mode and andrological evaluation of stallions treated with human corionic gonadotrofin $(h C G)$ in different seasons of the year]

\author{
R.C.L. Morais ${ }^{1}$, G.A. Dutra ${ }^{2}$, J.P. Oliveira ${ }^{3}$, F.C.V.L. Fonseca ${ }^{1}$, \\ C.F.P.M. Carvalho ${ }^{4}$, J.C.F. Jacob ${ }^{4}$
}

\author{
${ }^{1}$ Alunos de pós-graduação - Universidade Federal Rural do Rio de Janeiro - Seropédica, RJ \\ 2 Centro Universitário de Belo Horizonte, Belo Horizonte, MG \\ ${ }^{3}$ Médico Veterinário autônomo - Guaratinguetá, SP \\ ${ }^{4}$ Universidade Federal Rural do Rio de Janeiro - Seropédica, RJ
}

\begin{abstract}
RESUMO
Utilizaram-se quatro garanhões nos meses de janeiro, abril, julho e outubro de 2016, em dois protocolos, em que: GI ( $\mathrm{n}=4 ; 5 \mathrm{~mL}$ de solução salina, i.v.) e GII ( $\mathrm{n}=4 ; 5000 \mathrm{UI}$ de $\mathrm{hCG}$, i.v. $)$ e subdividiram-se esses protocolos em ciclos (C1 e C2), seguindo o esquema crossover, sendo: CI=animais 1 (GI) e 2 (GII), avaliados nos dias D1, D3 e D5, e animais 3 (GI) e 4 (GII), em D2, D4 e D6; CII= animais 1 (GII) e 2 (GI), em D1, D3, D5, e animais 3 (GII) e 4 (GI), em D2, D4 e D6. Realizou-se o tratamento em D1 e D2 de cada ciclo e a ultrassonografia testicular no modo color Doppler e Doppler espectral, uma hora antes de cada coleta de sêmen e imediatamente após. Avaliou-se: número de reflexo de Flehmen, de montas sem ereção, início da monta, tempo de reação à ereção e total da monta e análises de qualidade seminal. Estatisticamente foram utilizados os testes de qui-quadrado e ANOVA. Não houve diferenças estatísticas $(\mathrm{P}>0,05)$ entre os parâmetros analisados. Concluiu-se que uma única dose de 5000UI de hCG em garanhões não causou alterações significativas nos parâmetros avaliados em diferentes estações do ano.
\end{abstract}

Palavras-chave: andrologia, garanhões, hCG, ultrassom, Doppler

\begin{abstract}
Four stallions were used in January, April, July and October 2016 in two protocols: GI ( $n=4 ; 5 \mathrm{ml}$ saline, $i v)$ and $\operatorname{GII}(n=4 ; 5000 h C G, i v)$, and these protocols were subdivided into cycles (C1 and C2) following the cross over scheme, as follows: CI=animal 1 (GI) and 2 (GII) evaluated on days DI, D3 and D5 and animal 3 (GI) and 4 (GII) at D 2, D 4 and D 6; CII=animal 1 (GII) and 2 (GI) at D1, D3, D5 and animal 3 (GII) and 4 (GI) at D2, D4 and D6. Treatment was performed on D1 and D2 of each cycle and testicular ultrasound in color Doppler and spectral Doppler mode, one hour before each semen collection and immediately after. We evaluated: Flehmen's reflex number, mounts without erection, start of the mount, reaction time to erection and total mount and seminal quality analyzes. Statistically, the Chi-square and ANOVA tests were used. There were no statistical differences $(\mathrm{P}>0.05)$ between the analyzed parameters. It was concluded that a single dose of 5000IU hCG in stallions did not cause significant changes in the parameters evaluated in different seasons of the year.
\end{abstract}

Keywords: andrology, stallions, $h C G$, ultrasound, Doppler

Recebido em 8 de agosto de 2019

Aceito em 26 de dezembro de 2019

E-mail: morais.l.cassiavet@hotmail.com 


\section{INTRODUÇÃOO}

O conhecimento sobre a anatomia dos órgãos reprodutivos do garanhão e sua interação dos ecos com os tecidos são os primeiros passos para a interpretação do exame ultrassonográfico no exame andrológico. As ultrassonografias (US) no modo B e Doppler fornecem importantes informações sobre a arquitetura interna dos órgãos genitais e vascularização testicular, auxiliando no diagnóstico e melhorando o prognóstico de inúmeras patologias. No entanto, ainda hoje poucos médicos veterinários utilizam a US no exame andrológico devido à falta de conhecimento da anatomia ultrassonográfica e pouco treinamento especializado. Isso pode acarretar possíveis diagnósticos patológicos tardios, dificultando o tratamento e comprometendo a futura fertilidade do garanhão (Monteiro, 2017).

Segundo Lima et al. 2000, ao longo da estação de monta, pode ocorrer um decréscimo qualitativo em alguns parâmetros reprodutivos dos garanhões, como na libido e na qualidade espermática. A estimulação da esteroidogênese pelas células de Leydig é um parâmetro utilizado nos estudos das alterações reprodutivas ligadas à esfera hormonal em várias espécies e já se comprovou que o uso de gonadotrofina coriônica humana (hCG) tem um papel importante nessa estimulação da resposta esteroidogênica (Hall, 1994; Lima et al., 2000). O hCG tem sido usado para avaliar os receptores para o hormônio luteinizante (LH) em células de Leydig de equinos, com a vantagem de ser mais estável que o LH equino, sendo que ambas as gonadotrofinas estimulam a esteroidogênese (Hall,1994).

Diante dessas informações, esta pesquisa objetivou avaliar possíveis alterações nos parâmetros ultrassonográficos vasculares das gônadas, assim como mudanças nas características do comportamento reprodutivo no ato da monta e nos parâmetros seminais de garanhões férteis após a administração de hCG, durante as diferentes estações do ano.

\section{MATERIAL E MÉTODOS}

O estudo foi realizado na Universidade Federal Rural do Rio de Janeiro, situada no município de Seropédica- RJ, durante os meses de janeiro (verão), abril (outono), julho (inverno) e outubro (primavera) de 2016, sendo aprovado pela Comissão de Ética no Uso de Animais do Instituto de Medicina Veterinária da UFRRJ (Ceua/UFRRJ), protocolado sob o $\mathrm{n}^{\mathbf{0}}$ 9336110316. Foram utilizados quatro garanhões férteis, da raça Mangalarga Marchador, em idade reprodutiva (cinco-10 anos), que permaneceram alojados em baias, recebendo volumoso (2\% do peso vivo) e $4 \mathrm{~kg} /$ dia de ração balanceada, com acesso livre a água, sal mineral, e soltos em piquetes no período da tarde.

Os animais foram divididos em dois grupos: GI $(\mathrm{n}=4)$, sendo administrados $5 \mathrm{~mL}$ de solução salina, e GII $(n=4)$ 5000UI $(5 \mathrm{~mL})$ de hCG (Chorulon ${ }^{\circledR}$, MSD-Saúde Animal, Brasil), ambos i.v., no primeiro dia de cada ciclo. Em cada estação do ano, os protocolos foram subdivididos em dois ciclos ( $\mathrm{C} 1$ e $\mathrm{C} 2$ ), com seis dias cada e com um intervalo de três dias entre os ciclos, seguindose o esquema crossover, em que: $\mathrm{CI}=$ animais 1 (GI) e 2 (GII), avaliados nos dias D1, D3 e D5, e animais 3 (GI) e 4 (GII) em D2, D4 e D6; $\mathrm{CII}=$ animais 1 (GII) e 2 (GI), avaliados nos dias D1, D3, D5, e animais 3 (GII) e 4 (GI) em D2, D4 e D6.

A cada ciclo avaliado, os garanhões foram submetidos aos protocolos de tranquilização previamente às avaliações ultrassonográficas. Para realização desse exame, os animais eram mantidos em um brete de contenção para tranquilização com cloridrato de xilazina $2 \%$, na dose de 0,00028mg/ kg/i.v. Em seguida, foi realizada a US no modo color Doppler, para se avaliar a porcentagem de vascularização em ambos os testículos, e US Doppler espectral, para se mensurar o índice de resistividade (RI) das artérias testiculares. Esses procedimentos ocorreram uma hora antes e imediatamente após a coleta de sêmen.

Após a primeira US diária, os animais foram mantidos em um piquete, por uma hora, póstranquilização. Durante esse tempo, uma égua em estro foi contida, sempre no mesmo local, para realização da coleta de sêmen. Essas coletas foram filmadas para se avaliarem posteriormente as seguintes análises comportamentais: número de reflexos de Flehmen, número de montas sem ereção (MSE), início da monta com ereção (IME), tempo de reação à ereção (TER) e tempo total da monta (TTM). Terminada a coleta, o sêmen foi encaminhado ao laboratório para realização das 


\section{Ultrassonografia testicular...}

seguintes análises da qualidade seminal: mensuração do volume filtrado, motilidade, vigor, concentração, e o garanhão foi imediatamente reencaminhado ao brete de contenção para uma nova tranquilização e US.

A US foi realizada utilizando-se equipamento de ultrassom Doppler Mindray Z5VET e transdutor linear 75L50EAV, com emissão de onda de $5,0 / 7,5 \mathrm{MHz}$. O exame foi realizado em ambos os testículos e cordões espermáticos dos garanhões.

Após a identificação do parênquima testicular em modo B, a US color Doppler foi ativada, e o transdutor foi colocado, em relação a cada testículo, nos seguintes posicionamentos: craniovertical, caudovertical e látero-horizontal. A perfusão vascular foi estimada subjetivamente por meio da pontuação da extensão dos sinais coloridos para o fluxo sanguíneo em tempo real, em intervalo contínuo de um minuto: $0 \%$ (vascularização não aparente), $1 \%-25 \%$ (baixa), $25 \%-50 \%$ (intermediária), $50 \%-75 \%$ (alta), $75 \%-100 \%$ (muito alta) (Silva et al., 2005). Para cada varredura contínua de US color Doppler de um minuto, foram selecionadas três imagens fixas no momento de maior brilho da coloração. As médias de porcentagem de coloração das imagens de cada posicionamento foram utilizadas caracterizando-se o parênquima testicular como um todo.

Além da avaliação em US color Doppler, foram realizados escaneamentos em US Doppler espectral da artéria testicular no cordão espermático. $\mathrm{O}$ cordão espermático foi visualizado em US modo $\mathrm{B}$, por meio do transdutor na posição horizontal, bem próximo ao testículo. A US Doppler espectral foi ativada inserindo-se o cursor espectral sobre o ramo da artéria testicular ao nível do cordão espermático. Para os exames espectrais, a configuração das faixas de velocidade e o tamanho do portão espectral (gate) foram ajustados durante cada exame, para se obter uma sequência de gráficos Doppler espectral com ciclos cardíacos sistólicos e diastólicos simétricos e distintos, sem aliasing. As configurações de ganho, filtros e frequência de repetição de pulso $(\mathrm{PRF}=1)$ foram uniformes em todos os exames.

O espectro Doppler obtido foi registrado em vídeo e analisado posteriormente. Utilizaram-se três pulsos do ciclo cardíaco, sendo escolhido o central como representativo real da velocidade do fluxo sanguíneo expressado pelo RI (Silva et al., 2005) (Fig. 1).

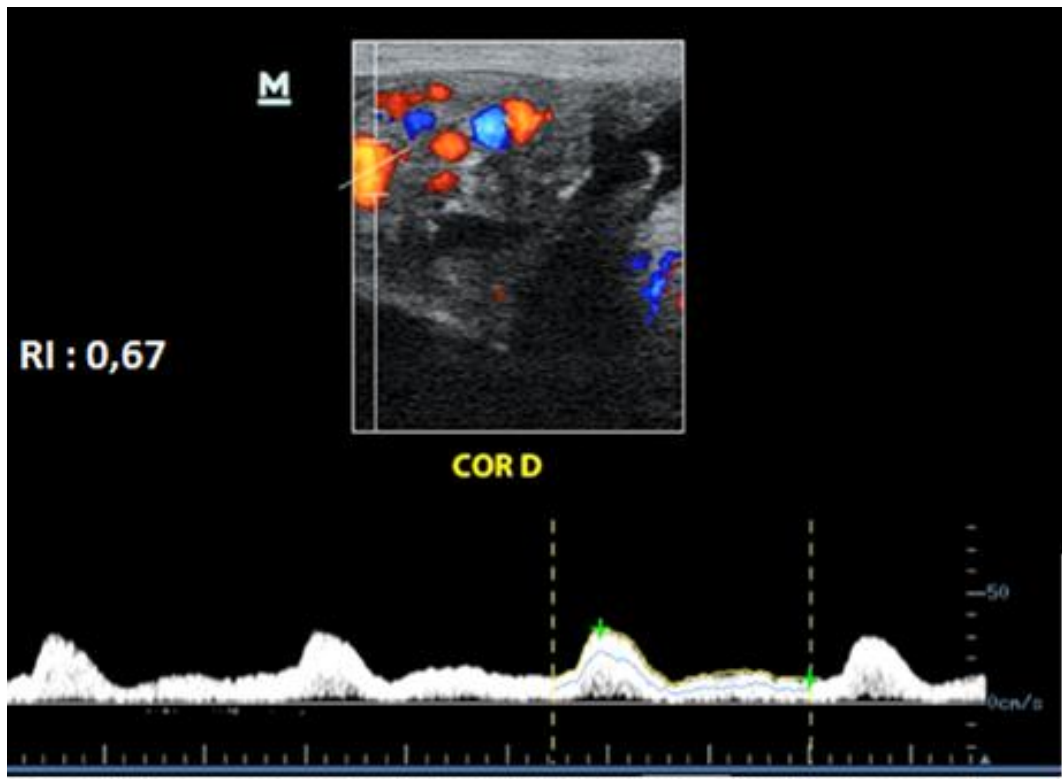

Figura 1. Ultrassonografia triplex (modo B, color Doppler e Doppler espectral). Cursor sobre ramo da artéria testicular do cordão espermático direito (Cor D) de garanhão e obtenção do espectro Doppler para medição da velocidade do fluxo sanguíneo e do índice de resistividade (RI). 
Todos os dados obtidos foram comparados entre os dias experimentais, entre os tratamentos e entre os meses pesquisados, utilizando-se o teste ANOVA e o teste de qui-quadrado, com nível de significância de $5 \%$.

\section{RESULTADOS E DISCUSSÃO}

Até o presente momento, não foram encontradas pesquisas sobre a vascularização testicular em equinos aferida por US color Doppler, mensurada por método subjetivo em porcentagem de coloração da imagem. No presente estudo, os resultados obtidos por essa técnica não sofreram alterações entre os tratamentos com solução salina e com hCG, como também não houve diferença antes e após as coletas de sêmen nem entre as estações do ano (Tab. 1).

Tabela 1. Média e desvio-padrão da coloração subjetiva testicular obtida por meio da ultrassonografia em modo color Doppler de garanhões tratados com solução salina ou gonadotrofina coriônica humana (hCG) nas diferentes estações do ano $(\mathrm{P}>0,05)$

\begin{tabular}{ccccc}
\hline & & \multicolumn{4}{c}{ Média geral de \% fluxo sanguíneo testicular em color Doppler } \\
\cline { 2 - 5 } Estação do ano & \multicolumn{2}{c}{ Grupo solução salina } & \multicolumn{2}{c}{ Grupo hCG } \\
& AC & DC & AC & DC \\
\hline Verão & $13,54 \pm 3,22$ & $14,8 \pm 3,44$ & $14,91 \pm 4,64$ & $16,09 \pm 4,39$ \\
Outono & $10,57 \pm 3,99$ & $11,64 \pm 3,33$ & $12,37 \pm 6,21$ & $15,18 \pm 4,74$ \\
Inverno & $17,82 \pm 6,0$ & $20,7 \pm 4,78$ & $19,18 \pm 6,15$ & $20,87 \pm 5,03$ \\
Primavera & $22,33 \pm 4,79$ & $26,23 \pm 4,71$ & $26,07 \pm 5,94$ & $28,74 \pm 4,78$ \\
\hline
\end{tabular}

* AC: antes da coleta de sêmen; DC: após a coleta de sêmen; hCG: gonadotrofina coriônica humana.

Utilizando-se a US Doppler espectral, a média geral de RI obtida na artéria testicular do cordão espermático nos garanhões durante todo o período experimental foi de $0,76 \pm 0,05$, estando esses valores próximos aos descritos por Pozor e McDonnell (2002), que obtiveram médias de RI $0,97(0,72-1,00)$ em animais não sedados previamente com cloridrato de xilazina $(0,5 \mathrm{mg} /$ $\mathrm{kg}$ /i.v.) e de RI 0,86 $(0,6$ - 1,00) com a sedação.
Não foram observadas diferenças estatísticas entre os valores de RI obtidos nos exames realizados entre os grupos GI e GII, antes e após as coletas de sêmen, nem entre as estações do ano. Os valores médios de RI encontrados para o GI e o GII antes e após as coletas de sêmen, durante as quatro estações do ano, encontram-se na Tab. 2.

Tabela 2. Média e desvio-padrão do índice de resistividade (RI) da artéria testicular de garanhões tratados com solução salina ou com gonadotrofina coriônica humana (hCG) nas diferentes estações do ano

\begin{tabular}{ccccc}
\hline \multirow{2}{*}{ Estações do ano } & \multicolumn{4}{c}{ Média geral do Índice de resistência RI (0-1) da artéria testicular } \\
\cline { 2 - 5 } & \multicolumn{2}{c}{ Grupo solução salina } & \multicolumn{2}{c}{ Grupo hCG } \\
& AC & DC & AC & DC \\
\hline Verão & $0,70 \pm 0,1$ & $0,77 \pm 0,08$ & $0,72 \pm 0,12$ & $0,73 \pm 0,13$ \\
Outono & $0,81 \pm 0,11$ & $0,80 \pm 0,12$ & $0,80 \pm 0,13$ & $0,76 \pm 0,13$ \\
Inverno & $0,78 \pm 0,1$ & $0,77 \pm 0,1$ & $0,73 \pm 0,11$ & $0,74 \pm 0,13$ \\
Primavera & $0,83 \pm 0,07$ & $0,80 \pm 0,04$ & $0,76 \pm 0,07$ & $0,72 \pm 0,08$ \\
\hline
\end{tabular}

*AC: antes da coleta de sêmen; DC: após a coleta de sêmen; hCG: gonadotrofina coriônica humana; RI: índice de resistividade.

Uma das poucas pesquisas que utilizaram a US Doppler espectral para caracterização do fluxo sanguíneo testicular em equinos após administração de hCG foi realizada por Pozor et al. (2006a). Os autores efetuaram tratamentos com 500UI, 1000UI, 2500UI e 6000UI de hCG e executaram a ultrassonografia antes do tratamento, uma hora, 72 horas e sete dias após. Os autores verificaram um aumento no tempo máximo de velocidade (TAM) e na taxa de fluxo sanguíneo arterial total $(\mathrm{TABFR}=$ fluxo sanguíneo arterial total/total testicular volume $\mathrm{x}$ 100) no exame realizado uma hora após o tratamento, com a dose de 6000UI de hCG, e não observaram nenhum efeito de qualquer dos tratamentos nesses parâmetros após sete dias. Apesar de Pozor et al. (2006a) terem avaliado outros parâmetros, como PI, RI, velocidade 
diastólica final (EDV), TAM, TABFR e fluxo sanguíneo arterial total $(\mathrm{TABF}=$ tempo médio da velocidade máxima $\mathrm{x} A$; sendo $\mathrm{A}=$ corte transversal da artéria), os autores citaram somente o aumento no TAM e no TABFR, dificultando comparações com o RI obtido no presente trabalho.

Cochard et al. (2000) citaram que, para mensurações do fluxo sanguíneo em US Doppler espectral, os valores de velocidade, como velocidade diastólica final (EDV), são variáveis e inconsistentes entre as aferições. Por esse motivo, no presente estudo, optou-se pela utilização do RI por apresentar pouca variação (0-1), além de ser um índice mais sensível e comumente utilizado na detecção de distúrbios do fluxo sanguíneo testiculares em humanos, de acordo com Biagiotti et al. (2002). Outra vantagem desse índice em relação à análise isolada de velocidade é de que os índices (RI e PI) têm a independência da correção do ângulo, sendo eles utilizados para avaliar vasos muito pequenos e tortuosos, onde é difícil a correção do ângulo (Mcdicken e Hoskins, 2014).

A respeito da sazonalidade, Pozor et al. (2006b), ao utilizarem US Doppler espectral em garanhões, observaram que ocorrem flutuações no fluxo sanguíneo da artéria testicular entre as diferentes estações e que essa variação pode estar relacionada também com as mudanças demonstradas no volume testicular e na produção de espermatozoides durante as estações. A presente pesquisa demonstrou que houve diferença entre as estações do ano para as seguintes variáveis: pico de velocidade sistólica (PSV), velocidade diastólica final (EDV), diâmetro arterial, tempo médio da velocidade máxima (TABF), concentração de testosterona e de estradiol.

Este estudo relatou que a perfusão testicular aumenta durante a estação reprodutiva, asseverando ser principalmente por aumento do diâmetro da artéria testicular e aumento da velocidade média do fluxo sanguíneo, portanto, as flutuações no fluxo sanguíneo testicular podem estar envolvidas na regulação das mudanças sazonais no volume testicular e na produção de espermatozoides. Contudo, os resultados de Pozor et al. (2006b) foram obtidos ao norte da linha do equador, em local de estações climáticas bem definidas, discordando dos resultados da presente pesquisa, em que não houve diferença significativa entre as estações do ano em relação ao RI. Esses resultados se justificam pelo fato de a região onde o estudo foi realizado não ter um fotoperíodo distinto entre as estações.

As manifestações comportamentais observadas nos garanhões durante as montas, como número de reflexo de Flehmen, início da monta com ereção (IME), tempo de reação à ereção (TER) e tempo total da monta (TTM), não apresentaram diferença significativa entre os grupos com administração de solução salina ou hCG nem entre as estações avaliadas (P>0,05) (Tab. 3).

Tabela 1. Média e desvio-padrão de parâmetros comportamentais no ato das coletas seminais dos garanhões tratados com gonadotrofina coriônica humana (hCG) ou solução salina nas diferentes estações do ano $(\mathrm{P}>0,05)$

\begin{tabular}{|c|c|c|c|c|c|c|c|c|}
\hline \multirow{4}{*}{ comportamento } & \multicolumn{8}{|c|}{ Estação do ano } \\
\hline & \multicolumn{2}{|c|}{ Verão } & \multicolumn{2}{|c|}{ Outono } & \multicolumn{2}{|c|}{ Inverno } & \multicolumn{2}{|c|}{ Primavera } \\
\hline & \multicolumn{8}{|c|}{ Grupos } \\
\hline & $\begin{array}{c}\text { Sol. } \\
\text { salina }\end{array}$ & hCG & $\begin{array}{l}\text { Sol. } \\
\text { salina }\end{array}$ & hCG & $\begin{array}{c}\text { Sol. } \\
\text { salina }\end{array}$ & hCG & Sol. salina & hCG \\
\hline R. de Flehmen $\left(\mathrm{n}^{\circ}\right)$ & $0,7 \pm 0,9$ & $0,3 \pm 0,5$ & $0,0 \pm 0,0$ & $0,0 \pm 0,0$ & $0,0 \pm 0,0$ & $0,0 \pm 0,0$ & $0,7 \pm 0,9$ & $0,7 \pm 0,9$ \\
\hline $\operatorname{MSE}\left(n^{o}\right)$ & $4,3 \pm 2,6$ & $3,7 \pm 1,7$ & $1,0 \pm 0,0$ & $0,0 \pm 0,9$ & $2,3 \pm 0,9$ & $2,3 \pm 0,9$ & $0,7 \pm 0,5$ & $0,7 \pm 0,5$ \\
\hline TRE (s) & $20,8 \pm 40,3$ & $24,4 \pm 42,3$ & $27,2 \pm 20,3$ & $20,2 \pm 10,2$ & $38,8 \pm 73,1$ & $35,3 \pm 41,9$ & $28,4 \pm 19,2$ & $14,3 \pm 11,1$ \\
\hline $\operatorname{IME}(\mathrm{s})$ & $48,1 \pm 76,8$ & $39,5 \pm 69,9$ & $71,1 \pm 44,2$ & $39,1 \pm 6,3$ & $58,8 \pm 67,6$ & $56,8 \pm 69$ & $35 \pm 19,3$ & $29,1 \pm 13,3$ \\
\hline TTM (s) & $35,7 \pm 27,1$ & $38,7 \pm 41,5$ & $33,2 \pm 6,9$ & $29,8 \pm 5,5$ & $36,3 \pm 41,8$ & $28,9 \pm 17,1$ & $29,7 \pm 6,7$ & $27,4 \pm 7,5$ \\
\hline
\end{tabular}

*Sol. salina $=$ solução salina; hCG $=$ gonadotrofina coriônica humana; $\mathrm{R}$. de Flehmen = reflexo de Flehmen; $\mathrm{N}^{\circ} \mathrm{MSE}=\mathrm{n}^{\circ}$ de monta sem ereção; TRE = tempo de reação à ereção; IME= início de monta com ereção; TTM: tempo total de monta. 
Os parâmetros médios de comportamento reprodutivo de garanhões da raça Mangalarga Marchador obtidos encontram-se dentro dos parâmetros descritos para a espécie equina por McDonnell (1992), os quais relatam a frequência de reflexo de Flehmen: 2 (0-10); tempo de reação à ereção (s): 10 (0-500); $n^{\circ}$ de montas: 1 (1-3); início de monta com ereção (s): 15 (8-20); e tempo total de monta (s): 20 (15-45).

Thompson et al. (1977) utilizaram garanhões submetidos ao tratamento com luz artificial simulando o aumento natural do fotoperíodo em uma região de latitude $40^{\circ} 35^{\prime}$ ao norte e não verificaram alterações em relação ao número de montas. No entanto, o tempo de reação à ereção (TER) foi menor nos animais que foram submetidos a aumento de luz. Esse fato já havia sido relatado por Pickett et al. (1976), os quais observaram que a média do TRE tende a ser a maior durante o inverno e menor durante a estação de monta natural. Porém, o presente estudo foi realizado nas proximidades da linha do equador, onde há pouca diferença entre a duração do fotoperíodo nas estações do ano. Esse fator, portanto, justifica a dificuldade na percepção da diferença comportamental nos garanhões avaliados por este experimento.

Em relação aos parâmetros seminais avaliados: volume filtrado, motilidade, vigor e concentração, os resultados obtidos não apresentaram diferenças entre os tratamentos com solução salina e com hCG, nem entre as estações do ano $(\mathrm{P}>0,05)$ (Tab. 4).

Tabela 4. Média e variação dos parâmetros seminais de garanhões tratados com gonadotrofina coriônica humana (hCG) ou solução salina nas diferentes estações do ano (P>0,05)

\begin{tabular}{ccccccccc} 
& \multicolumn{7}{c}{ Estação do ano } \\
\cline { 2 - 9 } $\begin{array}{c}\text { Parâmetros } \\
\text { seminais }\end{array}$ & \multicolumn{7}{c}{ Verão } & \multicolumn{7}{c}{ Outono } & \multicolumn{7}{c}{ Inverno } \\
\cline { 2 - 9 } & $\begin{array}{c}\text { Sol. } \\
\text { salina }\end{array}$ & hCG & $\begin{array}{c}\text { Sol. } \\
\text { salina }\end{array}$ & hCG & $\begin{array}{c}\text { Sol. } \\
\text { salina }\end{array}$ & hCG & $\begin{array}{c}\text { Sol. } \\
\text { salina }\end{array}$ & hCG \\
\hline $\begin{array}{c}\text { Vol. Filtrado } \\
(\mathrm{mL})\end{array}$ & $44,6 \pm 25$ & $48,8 \pm 33,8$ & $50 \pm 17$ & $55,4 \pm 15,7$ & $45,5 \pm 28,7$ & $42 \pm 21,1$ & $57,8 \pm 17,5$ & $55,3 \pm 11,1$ \\
$\begin{array}{c}\text { Motilidade } \\
(\%)\end{array}$ & $69,1 \pm 13$ & $60,8 \pm 17,7$ & $61,6 \pm 18,7$ & $70,5 \pm 7,6$ & $65,4 \pm 10,8$ & $69,1 \pm 11,3$ & $71,6 \pm 7,4$ & $68,9 \pm 12,6$ \\
$\begin{array}{c}\text { Vigor (0-5) } \\
\text { Concentração } \\
\left(\mathrm{x} 10^{6}\right)\end{array}$ & $3,2 \pm 0,6$ & $3 \pm 0,6$ & $3,3 \pm 0,8$ & $3,2 \pm 0,4$ & $3,2 \pm 0,4$ & $3,3 \pm 0,5$ & $3,1 \pm 0,3$ & $3,1 \pm 0,7$ \\
$*$ * & $62,3 \pm 36,3$ & $51,2 \pm 31,3$ & $53,7 \pm 32,5$ & $49,4 \pm 21,3$ & $209 \pm 181$ & $180 \pm 129,4$ & $155 \pm 37,66$ & $172,5 \pm 123,6$ \\
\hline hCG $=$ gonadotrofina coriônica humana
\end{tabular}

*hCG= gonadotrofina coriônica humana.

O volume de sêmen filtrado encontrado (de $42 \pm 21,1$ a $55,4 \pm 15,7 \mathrm{~mL})$ está entre os padrões desejáveis pelo Colégio Brasileiro de Reprodução Animal (Manual..., 2013), que variam de 40 a $60 \mathrm{~mL}$, e de acordo com os valores demonstrados por Rua et al. (2016), que também trabalharam com a raça Mangalarga Marchador, no estado do Rio de Janeiro, Brasil, e obtiveram volume de $44,4 \pm 27,9 \mathrm{~mL}$.

As porcentagens de motilidade seminal obtidas foram no verão: $69,1 \pm 13$ e $60,8 \pm 17,7$; outono: 61,6 $\pm 18,7$ e 70,5; inverno: $65,4 \pm 10,8$ e $69,1 \pm 11,3$; primavera: 71,6 $\pm 7,4$ e 68,9 $\pm 12,6$ (para GI e GII, respectivamente), estando de acordo com Freitas (2010), que, quando trabalhou com garanhões da raça Mangalarga Marchador, obteve valores médios para verão: $67,8 \pm 7,5$; outono:74,6 66,9 ; inverno:74,5 $\pm 5,9$; primavera: $66,4 \pm 8,9$. Pickett et al. (1976), no hemisfério norte, e Leme et al. (2012), no hemisfério sul, não observaram diferenças estacionais na motilidade espermática. Esses autores relataram que possíveis diferenças estacionais na motilidade demonstradas entre diversos estudos podem ser influenciadas pelo manejo dos animais, pela frequência na coleta de sêmen e pelas condições ambientais, como temperatura e umidade.

Assim como a motilidade, o vigor espermático também não diferiu entre os grupos e entre as estações estudadas, sendo obtidos os seguintes valores, para GI e GII, respectivamente: primavera: $3,1 \pm 0,3$ e $3,1 \pm 0,7$; verão: $3,2 \pm 0,6$ e $3 \pm 0,6$; outono: $3,3 \pm 0,8$ e $3,2 \pm 0,4$; inverno: $3,2 \pm 0,4$ e $3,3 \pm 0,5$. Os dados obtidos corroboram os valores encontrados no estado do Rio de Janeiro, Brasil, que foram relatados por Rua e 
colaboradores (2016), os quais obtiveram uma

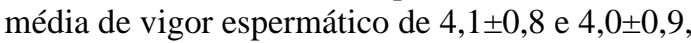
dentro e fora da estação reprodutiva, respectivamente (sem diferença significativa). Além disso, estão de acordo com dados relatados no hemisfério norte, por Pickett et al. (1998), onde a motilidade progressiva e o vigor espermático não pareceram sofrer influência da estação do ano.

Os valores das concentrações espermáticas (milhões de espermatozoides/mL), com administração de solução salina ou hCG, respectivamente, foram de: primavera: $155 \pm 37,66$ e $172,5 \pm 123,6$; verão: $62,3 \pm 36,3$ e $51,2 \pm 31,3$; outono: $53,7 \pm 32,5$ e $49,4 \pm 21,3$; inverno: $209,1 \pm 181$ e $180,4 \pm 129,4$. Os resultados obtidos foram semelhantes aos de Robalo Silva et al. (2007), em Portugal, que observaram na primavera: $138,7 \pm 16,9$; no verão: $151 \pm 16,5$; no

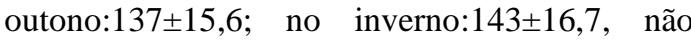
observando diferenças entre os ejaculados nas distintas estações do ano.

Entretanto, em estudos realizados no hemisfério sul, por Rua et al. (2016), foram relatadas diferenças dos valores de concentração espermática entre a estação reprodutiva $(507 \pm 615,9)$ e fora da estação $(278 \pm 224,9)$. Diferentemente, Freitas (2010), quando trabalhou com cavalos da raça Mangalarga Marchador, obteve maior média de concentração espermática no outono: $311,8 \pm 148,3$ e no inverno: $358,7 \pm 180,6$ do que na primavera: $178,9 \pm 97,0$ e no verão: $191,0 \pm 110,1$, fato que não foi observado no presente estudo. Freitas (2010) justificou as diferenças observadas na concentração espermática ao fato de o volume ejaculado ter sido maior durante a estação de monta.

Segundo Sieme et al. (2004), um fator importante que pode explicar a divergência nos resultados da concentração espermática é que esta pode variar, dependendo do intervalo entre coletas. Tal condição pode sugerir que esse é um dos fatores mais determinantes para as diferenças observadas entre estudos.

A influência da estação do ano na qualidade espermática de garanhões já foi caracterizada por alguns autores (Blottner et al., 2001; Janett et al., 2003), que evidenciaram a obtenção da capacidade reprodutiva máxima ocorrendo nos meses de primavera e verão em países com marcada diferença climática. Entretanto, essa influência não tem sido verificada em garanhões que vivem em regiões de clima tropical (Leme et al., 2012), fato que confirma os resultados encontrados no presente experimento.

Existem evidências substanciais de que os ciclos reprodutivos possam ser gerados de forma endógena, sem nenhum sinal de mudança anual dos fatores ambientais (Turek e Van Cauter, 1994), e, nos trópicos, é menos provável que a regulação sazonal da atividade reprodutiva seja baseada na alteração do fotoperíodo em regiões abaixo de $30^{\circ}$ de latitude da linha do equador (Bronson e Heideman, 1994), fato que concorda com os resultados obtidos na presente pesquisa, que foi realizada em latitude de $22^{\circ} 45^{\prime} 48.74^{\prime \prime} \mathrm{S}$.

Vale ressaltar que a América do Sul é afetada pelas oscilações causadas pelo efeito El Niño, que tornam ainda mais diversificadas e imprevisíveis as condições climáticas, podendo promover alterações relacionadas à reprodução (Leme et al., 2012).

\section{CONCLUSÃO}

A administração de uma única dose de 5000UI de hCG em garanhões da raça Mangalarga Marchador nas diferentes estações do ano não foi suficiente para causar alterações no fluxo sanguíneo dos testículos mensurados pelo método subjetivo em color Doppler e da artéria testicular do cordão espermático, mensurado pelo índice Doppler de resistividade. Também não ocasionou alterações no comportamento reprodutivo e nos parâmetros qualitativos e quantitativos do sêmen nas condições experimentais testadas.

\section{AGRADECIMENTO}

Conselho Nacional de Desenvolvimento Científico e Tecnológico (CNPq).

\section{REFERÊNCIAS}

BIAGIOTTI, G.; CAVALINI, G.; MODENINI, F.; VITALI, G. et al. Spermatogenesis and spectral echo-colour Doppler traces from the main testicular artery. BJU Int., v.90, p.903-908, 2002.

BLOTTNER, S.; WARNKER, C.; TUCHESCHERER, A. et al. Morphological and and functional changes of stallion spermatozoa after cryopreservation during breeding and non-breeding season. Anim. Reprod. Sci., v.65, p.75-88, 2001. 
BRONSON, F.H.; HEIDEMAN, P.D. Seasonal regulation of reproduction in mammals. In: KNOBIL, E.; NEILL, J.D. (Eds.). The physiology of reproduction. 2.ed. New York: Raven Press, 1994. $3302 \mathrm{p}$.

COCHARD, T.; TOAL, R.; SAXTON, A.M. Doppler ultrasonography features of thoracic limb arteries in clinically normal horses. Am. J. Vet. Res., v.61, p.183-190, 2000.

FREITAS, B.W. Parâmetros fisiológicos e seminais de garanhões da raça Mangalarga Marchador na região da zona da mata mineira no decorrer das estações climáticas. 2010. 72f. Dissertação (Mestrado em Medicina Veterinária) - Universidade Federal de Viçosa, Viçosa, MG.

HALL, P.F. Testicular steroid synthesis: organization and regulation. In: KNOBIL, E.; NEILL, J.D. (Eds.). The physiology of reproduction. 2 ed. New York: Raven Press, 1994. v.2, p.363-410.

JANETT, F.; THUN, R.; NIEDERER, K.; BURGER, D. et al. Seasonal changes in semen quality and freezability in the Warmblood stallion. Theriogenology, v.60, p.453-461, 2003.

LEME, D.P.; PAPA, F.O.; ROSER, J.F. Reproductive characteristics of stallions during the breeding and non-breeding season in a tropical region. Trop. Anim. Health Prod., v.44, p.17031707, 2012.

LIMA, S.B.; WISCHRAL, A.; FERREIRA, F.; VERRESCHI, I.T.N. Resposta esteroidogênica induzida por hCG em garanhões jovens Mangalarga: testosterona e sulfato de estrona plasmáticos. Braz. J. Vet. Res. Anim. Sci., v.37, p.52-55, 2000.

MANUAL de exame andrológico e avaliação de sêmen animal. 3ed. Belo Horizonte, CBRA, 2013. $104 \mathrm{p}$.

MCDICKEN, W.N.; HOSKINS, P.R.; Physics: principles, practise and artefacts. In: POZNIAK M.A.; ALLAN P.L. Clinical Doppler ultrasound. 3.ed. China: Elsevier, 2014. p.1-24.

McDONNELL, S.M. Normal and abnormal sexual behavior. Vet. N. Am. Equine Pract., v.8, p.71-89, 1992.

MONTEIRO, G.A. Ultrassonografia aplicada ao exame andrológico em garanhões. Rev. Bras. Reprod. Anim., v.41, p.157-168, 2017.

PICKETT, B.W.; FAULKNER, L.C.; SEIDEL JR, G.E.; BERNDTSON, W.E. et al. Reproductive physiology of stallion. 6: Seminal and behavior characteristics. J. Am. Vet. Assoc., v.43, p.617-625, 1976.
PICKETT, B.W.; VOSS, J.L.; CLAY, C.M. Managment of shuttle stallions for maximun reproductive efficiency - part 2. J. Equine Vet. Sci., v.18, p.280-287, 1998.

POZOR, M.A.; MACPHERSON, M.L.; TROEDSON, M.H.T.; VERSTEGEN, J. Effect of a single administration of human chorionic gonadotropin (hCG) on testicular blood flow in stallions. Anim. Reprod. Sci., v.94. p.146-147, $2006 \mathrm{a}$.

POZOR, M.A.; MACPHERSON, M.L.; TROEDSON, M.H.T.; VERSTEGEN, J. Effect of seasonality on testicular blood flow in mature stallions. Anim. Reprod. Sci., v.94, p.144-145, $2006 b$.

POZOR, M.A.; MCDONNEL, S.M. Doppler ultrassound measures of testicular blood flow in stallions. Theriogenology, v.58, p.437-440, 2002.

ROBALO SILVA, J.; AGRÍCOLA, R.; BARBOSA, M.; COSTA, L.L. Variação sazonal do volume testicular, da produção e qualidade do sêmen e do comportamento sexual de cavalos Lusitanos. Rev. Port. Cienc. Vet., v.102, p.119-125, 2007.

RUA, M.A.S.; QUIRINO, C.R.; BARTHOLAZZI JUNIOR, A.; NASCIMENTO, P.S. et al. Repetibilidade das características seminais, espermáticas e fertilidade de garanhões. Rev. Bras. Cienc. Agrar., v.11, p.124-131, 2016.

SIEME, H.; KATILA, T.; KLUG, E. Effect of semen collection practices on sperm characteristics before and after storage and fertility of stallions. Theriogenology, v.61, p.769-784, 2004.

SILVA, L.A.; GASTAL, E.L.; BEG, M.A.; GINTHER, O.J. Changes in vascular perfusion of the endometrium in association with changes in location of the embryonic vesicle in mares. Biol. Reprod., v.72, p.755-761, 2005.

THOMPSON JR, D.L.; PICKETT.; W.E. BERNDTSON.; J.L. et al. Reproductive physiology of the stallion. VIII. Artificial photoperiod, collection interval and seminal characteristics, sexual behavior and concentration of LH and testosterone in serum. J. Anim. Sci., v.44, p.656, 1977.

TUREK, F.W.; VAN CAUTER, E. Rhythms in reproduction. In: KNOBILL, E.; NEILL, J.D. (Eds.). The physiology of reproduction. 2.ed. New York: Raven Press. p.519-22, 1994. 